\title{
Influence of the milling parameters on the mechanical work intensity in planetary mills
}

\author{
F. J. Gotor ${ }^{1}$, M. Achimovicova ${ }^{2}$, C. Real ${ }^{1}$ and P. Balaz ${ }^{2}$ \\ ${ }^{1}$ Instituto de Ciencia de Materiales de Sevilla (CSIC-US), Américo Vespucio 49, 41092 \\ Sevilla, SPAIN \\ ${ }^{2}$ Institute of Geotechnics, Slovak Academy of Sciences, Watsonova 45, 04353 Košice, \\ SLOVAKIA
}

\begin{abstract}
The formation of ZnSe via a mechanically-induced self-sustaining reaction (MSR) from a $\mathrm{Zn} / \mathrm{Se}$ mixture showed that only size reduction and mixing of the reactants without product formation occurred during the induction period prior to ignition. Therefore, all mechanical energy supplied by the planetary mill during this time, called the ignition time $\left(t_{\mathrm{ig}}\right)$, was used exclusively in the activation of the reactants. This system was chosen to study the dependence of $t_{\text {ig }}$ on the main parameters characterising the milling intensity of planetary mills. The variation of the ignition time with the process conditions reflected changes in the mechanical dose rate of the planetary mill. A direct relationship between the inverse of the ignition time and the power of the planetary mill was established, which allows the validation of theoretical models proposed in the literature for the energy transfer in milling devices and the comparison of milling equipment efficiencies.
\end{abstract}

Keywords: Mechanochemistry; Self-sustaining Reaction; Ignition Time; Milling Energy; Efficiency; Scale-up 


\section{Introduction}

Milling is a significant unit operation in the many fields such as chemistry, pharmacy, mineral processing, and materials science. Many types of mills are employed in these fields and are ideally suited for wet or dry grinding processes, size reduction and dispersion, and deflocculation in solid-liquid systems [1-3]. When a chemical reaction is produced during milling, the process is commonly referred to as mechanochemistry, and the mill device must be considered a reactor. Mechanochemistry is generally performed in high-energy ball mills using powder reactant mixtures. During milling, the intimate mixing of reactants and the continuous creation of fresh interfaces and defects enable the gradual progression of solid state reactions at room temperature.

In many highly exothermic powder mixtures [4,5], ball milling can generate mechanically induced self-sustaining reactions (MSRs). An MSR process begins with an activation period, during which size reduction, mixing, and defect formation take place. At a given milling time, called the ignition time $\left(\mathrm{t}_{\mathrm{ig}}\right)$, a self-sustaining reaction is initiated and a reaction front propagates through the powder charge inside the vial. The ignition time depends on the experimental conditions and the chemical system under investigation [6]. MSR processes are comparable with self-propagating high-temperature synthesis (SHS), and they have similar requirements regarding the self-heating capacity of the mixtures.

The study of MSR processes is of fundamental importance because they can provide valuable information about the energy supplied by the milling devices during milling operations and the mechanisms of mechanochemical reactions [6]. MSR, in comparison to other mechanochemical processes, has the advantage of ignition time. This reproducible parameter can be measured by detecting the abrupt temperature increase of the milling vial [7] or the total pressure inside the vial [4] as a consequence of the heat released from the highly exothermic reaction. Ignition is a key moment in MSR that corresponds to a well defined 
critical state of the powder reactants that is reached when the mill device has provided the net amount of energy necessary to generate a self-sustaining reaction. This mechanical energy per unit mass needed to ignite a specific reactant mixture can be regarded as constant; therefore, the ignition time can be used as a reference point because its variation with process conditions reflects changes in the mechanical dose rate of the mill. This parameter also facilitates the comparison of reaction kinetics data obtained using different milling equipment and, consequently, their efficiencies [8].

Since the original work of Schaffer and McCormick [9], few works have focused on systematic studies of the effects of experimental milling conditions on the ignition time of MSR processes, and in most cases, the ball-to-powder ratio (BPR) was the only varied parameter [10-12]. Moreover, other studies have focused on the variation of the ignition time as a function of the composition of the reactant mixtures under otherwise identical experimental conditions $[13,14]$. However, all of these studies were performed in SPEX shaker mills because ignition time can be easily determined from the temperature profile measured on the outer surface of the vial by attaching a thermocouple. This is not possible in planetary mills because the motion of the vial results in breakage of the thermocouple.

Tschaharov et al. conducted the first systematic investigation on MSR studying the formation of metal chalcogenides (mainly sulphides) from their constituent elements [15]. They used a low energy vibratory mill; as a result, the observed ignition times were relatively long (up to $365 \mathrm{~min}$ for $\mathrm{ZnS}$ and $312 \mathrm{~min}$ for $\mathrm{ZnSe}$ ). With respect to selenides, Susol [16] observed MSR effects in Mg-Se, Sb-Se, and Bi-Se mixtures using a shaker mill. Although some authors [17-21] have recently reported the formation of ZnSe by high-energy ball milling of elemental mixtures of $\mathrm{Zn}$ and $\mathrm{Se}$, the occurrence of an MSR effect was not indicated because the milling products were generally inspected after long milling times, after ignition was likely to have occurred. However, in references 17 and 21, XRD diagrams of 
milled powders suggest that a self-sustaining reaction took place during milling in planetary mills after approximately 12 to $18 \mathrm{~min}$.

In this work, we report on $\mathrm{ZnSe}$ formation by an MSR process during milling in a planetary ball mill that has been adapted for determining the ignition time by measuring the pressure inside the vial. The influence of different milling parameters, such as the spinning rate, the charge of reactants, the number and type of balls, and the BPR on the ignition time was addressed. This work deals with the dynamics and efficiency of planetary mills and their dependence on milling parameters, irrespective of the specific phenomena occurring during the mechanochemical process. This study enabled a critical analysis of the models proposed in the literature to describe the energy transferred by planetary mills during operation by comparing our results with the theoretical predictions based on these models.

\section{Experimental procedure}

Zinc powder (99\% pure, $<325$ mesh, Strem Chemicals) and selenium powder $(<325$ mesh, Alfa Aesar) were milled under helium gas (Air Liquide) for the synthesis of ZnSe by an MSR process using a modified planetary ball mill (Micro Mill Pulverisette 7, Fritsch), allowing operation at a constant gas pressure and the detection of self-sustaining reactions during milling (Figure 1). This was enabled by connecting the vial to a gas cylinder by a rotating union (model 1005-163-038, Deublin) (Figure 1a) and a flexible polyamide tube (Figure 1b) and continuously monitoring the helium pressure with an SMC solenoid valve (model EVT307-5DO-01F-Q, SMC Co.) (Figure 1c) connected to a data acquisition system (ADAM-4000 series, Esis Pty Ltd.) (Figure 1d). At ignition, the exothermic reaction provoked an instantaneous temperature driven increase in the total pressure of the system that resulted in the appearance of a pressure spike in the time-pressure record, from which the ignition time of the process was obtained (Figure 1e). 
To explore the dependence of the ignition time on the milling conditions, different milling experiments were carried out by modifying the following parameters: the rotational speed of the planetary mill or spinning rate from 200 to $800 \mathrm{rpm}$ (in the Pulverisette 7 mill, the ratio between the rotational speed of the supporting disc and the rotational speed of the vial in the opposite direction is -1$)$, the powder charge from 3 to $9 \mathrm{~g}$, the type of balls (15 $\mathrm{mm}$ WC, $15 \mathrm{~mm}$ steel, $15 \mathrm{~mm} \mathrm{Si}_{3} \mathrm{~N}_{4}$, and $10 \mathrm{~mm}$ steel balls) and the number of balls from 3 to 7 for the $15 \mathrm{~mm}$ balls. In each milling experiment, the powder charge ( $\mathrm{Zn}$ and Se powders in a 1:1 stoichiometric ratio) and the milling balls were placed in a $45 \mathrm{~mL}$ tempered steel vial (67 HRC) and were ball-milled under 6 bars of high-purity helium gas. The vial was purged with helium several times, and the pressure was selected prior to milling.

X-ray powder diffraction diagrams were obtained with a Panalytical X'Pert Pro instrument (Eindhoven, The Netherlands) equipped with a $\theta / \theta$ goniometer, $\mathrm{Cu} \mathrm{K} \alpha$ radiation source $(40 \mathrm{kV}, 40 \mathrm{~mA})$, secondary $\mathrm{K}_{\beta}$ filter, and an $\mathrm{X}^{\prime}$ Celerator detector. The diffraction diagrams were scanned from $20^{\circ}$ to $80^{\circ}(2 \theta)$ in step-scan mode with a step size of $0.05^{\circ}$ and a counting time of $80 \mathrm{~s} / \mathrm{step}$.

The DSC experiments were conducted with a Setaram DSC 111 instrument with a sensitivity of $1 \mathrm{mV} / \mathrm{s}$. A flow of helium gas of $20 \mathrm{ml} / \mathrm{min}$, a heating rate of $10{ }^{\circ} \mathrm{C} / \mathrm{min}$ from room temperature to $800{ }^{\circ} \mathrm{C}$ and $25 \mathrm{mg}$ of powder were always used.

\section{Results and discussion}

\subsection{Formation of ZnSe by MSR}

Preliminary milling experiments were performed in order to provide information about the mechanochemical synthesis of $\mathrm{ZnSe}$ and confirm the appearance of an MSR process. Five grams of an equiatomic mixture of $\mathrm{Zn}$ and Se and seven steel balls with a diameter of $15 \mathrm{~mm}$ $(\mathrm{m}=13.56 \mathrm{~g})$, equivalent to a BPR value of 18.98 , were placed in the vial (about $1 / 3$ filling) 
and submitted to milling at $500 \mathrm{rpm}$ in a helium atmosphere. The milling was stopped at several incremental times (every $3 \mathrm{~min}$ ), and a small amount of the powder mixture was taken for examination by XRD.

Figure 2 displays the XRD diagram of the evolution of this $\mathrm{Zn} / \mathrm{Se}$ mixture with milling time. The XRD diagram at 0 min corresponds to the starting mixture, and the diagram at 14 min corresponds to the product obtained after ignition of the self-sustaining reaction without further milling. Our experimental device was able to stop milling at the precise instant that the pressure spike occurred (MSR effect) because of a controller connected to the solenoid valve, which activated an internal switch, resulting in the ON/OFF action of the planetary mill.

Figure 2 clearly shows that almost no chemical interaction between the Se and $\mathrm{Zn}$ reactants occurred during milling until the self-sustaining reaction was induced after 14 min of treatment. During milling prior to ignition, a decrease in the reflection intensity of the reactants was observed. This decrease was more pronounced in the reflections from Se, as a result of the capacity of high-energy ball milling to induce selenium amorphisation because of its special molecular structure that consists of helical molecular chains that are bonded together by weak van der Waals forces [22]. The XRD diagram after 12 min of milling indicates that only an insignificant amount of $\mathrm{ZnSe}$ was formed by diffusion before ignition. After ignition, Figure 2 indicates the near disappearance of the $\mathrm{Se}$ and $\mathrm{Zn}$ peaks, demonstrating that the self-sustaining reaction caused the instantaneous transformation of the reactants into the main final product, the cubic ZnSe phase. A small amount of hexagonal ZnSe was also detected, as well as unreacted zinc.

DSC measurements for the same Zn/Se mixtures shown in Figure 2 were conducted, and the results are shown in Figure 3. The DSC trace of the unmilled mixture clearly shows the endothermic peaks corresponding to the melting of selenium and zinc at $\sim 217{ }^{\circ} \mathrm{C}$ and 413 ${ }^{\circ} \mathrm{C}$, respectively. The strong exothermic signal extending over a wide temperature range (465 - 
$650{ }^{\circ} \mathrm{C}$ ) is associated with the formation of $\mathrm{ZnSe}$ from the $\mathrm{Zn}$-Se melt. Integrating both endothermic peaks, the calculated values for the heat of fusion of $\mathrm{Se}$ and $\mathrm{Zn}$ are approximately 5.77 and $7.80 \mathrm{kJmol}^{-1}$, respectively, which are in accordance with the literature values [23]. With increasing milling time, the same thermal events were detected up to ignition, where no DSC signal was observed, confirming the completion of the ZnSe forming reaction. The calculated heat of fusion of Se for the milled mixtures was only slightly lower, corroborating the negligible formation of $\mathrm{ZnSe}$ during milling prior to ignition. Figure 3 shows that the strong exothermic peak associated with the formation of $\mathrm{ZnSe}$ shifted towards lower temperatures with increasing milling time as a direct consequence of the activation of the powder mixture. For the sample milled for $12 \mathrm{~min}$, the formation of $\mathrm{ZnSe}$ started before the zinc was completely melted. For this reason, the heat of fusion of $\mathrm{Zn}$ that was calculated for this sample decreased to a greater extent than the value of the heat of fusion of Se obtained for the same mixture.

The XRD and DSC results indicate that during the induction period before ignition, milling mainly led to the mixing of reactants, size reduction and creation of lattice defects without product formation; therefore, it can be assumed that all of the mechanical energy provided by the planetary mill to the powder mixture was used exclusively in the activation of the reactants to reach the critical state that caused their ignition. Accordingly, taking as a reference the ignition time, i.e., the time required to attain this critical state and assuming that for the $\mathrm{Zn} / \mathrm{Se}$ mixture, this critical state was reached when an exact energy dose was provided, this reaction is appropriate to study how $t_{\text {ig }}$ and, therefore, the power supplied by the planetary mill depends on the main milling parameters. Moreover, as the ignition in this system was observed after a few minutes of milling, the experimental parameters could be varied over a wide range because ignition always took place after convenient milling times. 


\subsection{Dependence of $t_{\text {ig }}$ on milling parameters}

\subsubsection{Spinning rate}

The first set of experiments was performed in order to investigate the effects of the spinning rate of the planetary mill on the ignition time of the $\mathrm{Zn} / \mathrm{Se}$ reactant mixture (Figure 4a). For all of the experiments, seven WC balls with a diameter of $15 \mathrm{~mm}(\mathrm{~m}=26.39 \mathrm{~g})$ and 5 $\mathrm{g}$ of powder charge were employed. Figure $4 \mathrm{a}$ shows that $\mathrm{t}_{\mathrm{ig}}$ decreased as the spinning rate increased. A very short ignition time of 85 seconds was found for the maximum spinning rate of the mill, whereas a long time of 3.5 hours was observed at the lowest rate used. This trend shows that the product can be obtained by MSR even at a very low spinning rate because the continuous milling in a regime of low intensity does not cause the formation of $\mathrm{ZnSe}$ by the classical diffusion process that could act as an inert inhibitor to ignition.

The strong dependence observed between the ignition time and the spinning rate is an expected result because the spinning rate can be considered the most important parameter concerning the energy transferred to the powder charge in a planetary mill because it determines both the frequency and intensity of ball collisions. It has been proposed, based on a collision model [24-26], that the power supplied by a planetary mill is proportional to the third power of the spinning rate. From the data in Figure 4a, the same third power dependence between the inverse of the ignition time and the spinning rate $\left(1 / t_{i g} \propto\right.$ spinning rate $\left.{ }^{3}\right)$ can be obtained, as clearly shown by the log-log plot in Figure $4 \mathrm{~b}$. This result confirms that the variation of the ignition time with the experimental conditions reveals variations in the mechanical dose rate delivered by the planetary mill, and a direct relationship between $1 / t_{\mathrm{ig}}$ and the power transferred from the mill to the powder charge can be assumed.

\subsubsection{Powder charge.}


The BPR is also an important parameter directly related to the milling intensity, and it has a significant effect on the time required to reach a particular state. This parameter can be varied by changing the powder charge or the number and type of balls. Both effects were studied separately, and a second set of experiments was performed in which only the powder charge was varied and all of the other parameters were held constant. Seven steel balls with a diameter of $15 \mathrm{~mm}(\mathrm{~m}=13.56 \mathrm{~g})$, a spinning rate of $500 \mathrm{rpm}$ and powder charges of $3,5,7$, and $9 \mathrm{~g}$ were used.

Figure 5 shows the dependence of $t_{i g}$ on the powder charge, which appears to be perfectly linear. For this set of experiments, the frequency and intensity of collisions were maintained constant and, therefore, the linear behaviour observed implies that the energy absorbed by a unit mass of powder charge and supplied by the mill to induce ignition was also a constant value. This linearity suggest that the activation of the reactant mixture took place uniformly over the whole sample and allowed the definition of an ignition time per unit of mass of $176 \mathrm{sg}^{-1}$ for the experimental conditions employed in this set of tests. This feature provides clear evidence of the scalable character of milling experiments performed in planetary mills.

\subsubsection{Type and number of balls.}

In the third set of experiments, the type and number of balls were the only parameters varied. Three types of $15 \mathrm{~mm}$ balls were employed: $\mathrm{WC}$, steel, and $\mathrm{Si}_{3} \mathrm{~N}_{4}$, and the number of balls was varied from 3 to 7 for each type of ball. A powder charge of $5 \mathrm{~g}$ and a spinning rate of $500 \mathrm{rpm}$ were always used. The dependences of $t_{\mathrm{ig}}$ on the number and type of balls are illustrated in Figure 6, which shows that the three types of balls behaved similarly with the varying number of balls. When the number of balls was reduced, the number of collisions was 
also reduced and, consequently, the time needed to induce ignition was increased. We will see later that for 3 balls, the number of collisions is not the only parameter to consider.

The use of more than five balls only produced a slight decrease in ignition time, despite the expected increase in the number of collisions. This suggests that even if the number of collisions increased, a decrease in their efficiency, i.e., the energy transmitted per collision, must have occurred. The filling of the vial is also a parameter that must be considered. If there is not enough space for the balls to move freely inside the vial, the number of collisions between balls increases at the expense of the number of ball-wall collisions, which more efficiently transfer energy to the powder mixture.

Figure 6 also shows that the curves for the different types of balls increase toward longer ignition times with decreasing ball density. If the spinning rate, the powder charge and the number of balls were held constant, the only difference between the experiments carried out with the three types of balls was the intensity of collisions, as a direct consequence of the different ball mass and the resulting difference in kinetic energy. For this reason, the experiments with the heaviest balls (WC) had the shortest ignition times. For the region corresponding to between 5 and 7 balls, an inverse relationship between the ratio of densities and ignition times $\left(\rho_{1} / \rho_{2} \approx t_{i g}{ }^{2} / t_{i g}{ }^{l}\right)$ was found.

\subsubsection{Ball-to-powder ratio (BPR).}

There is a general consensus that the milling intensity is sufficiently characterised by the frequency of movement of the mill and the BPR ratio employed, regardless of the number, material, and size of the balls and the powder mass processed. For this reason, quite often only these two parameters are specified in milling studies. However, Figures $\mathbf{5}$ and $\mathbf{6}$ show that the effect on the ignition time produced by varying the BPR by modifying the powder charge or the number and nature of balls seems to be different. The values shown in Figures $\mathbf{5}$ 
and 6 were plotted together in Figure 7a, which shows the dependence of the ignition time on the BPR, regardless of how it was modified. This figure also incorporates two additional milling experiments performed with $10 \mathrm{~mm}$ steel balls containing 23 and 31 balls, respectively, and $5 \mathrm{~g}$ of powder charge.

Figure 7a shows that most of the data that corresponds to BPR values between 5 and 40 with adequate filling of the vials, as is generally employed by practitioners of ball milling experiments at a laboratory scale, follows an inverse relationship between the ignition time and the BPR $\left(1 / t_{i g} \propto B P R\right)$. It was found that higher values of the BPR produced shorter $\mathrm{t}_{\mathrm{ig}}$, independent of the nature of the milling medium. This inverse relationship is equivalent to a direct dependence on the powder-to-ball ratio (PBR) $\left(t_{i g} \propto P B R\right)$, as shown in Figure $7 \mathrm{~b}$. Although Figure 7 demonstrates that the BPR can characterise the milling intensity under the experimental conditions generally used, the number, material, size of balls, and mass of the processed powder should always be specified for an accurate and unambiguous comparison of milling experiments conducted in different laboratories.

Figures $7 \mathrm{a}$ and $7 \mathrm{~b}$ also show that the milling experiments performed with three balls do not obey the established trend, with extremely long ignition times when compared to processes with similar BPR values and carried out with a higher number of balls. The apparent deviation seems to begin with processes using 4 balls. This deviation occurs because in the case of three balls, there are no collisions during the milling treatment because the balls are not launched toward the opposite wall of the vial and, in fact, the balls slide on the walls. This was simply proved because the characteristic shrill sound of collisions is not heard during milling [27]. Because the balls slide, the energy supplied to the powder is primarily transferred by friction, which is much less effective than collisions. Therefore, the ignition time increases significantly. With 4 balls, collisions and friction occurred simultaneously. It is 
worth noting that the ignition of the mixture can also be induced by friction alone, which demonstrates that $\mathrm{ZnSe}$ is an ideal system to perform this kind of study.

\subsection{Assessment of theoretical models describing the energy transfer in planetary mills.}

The characterisation of the actual intensity of a milling treatment is a difficult task. Several modelling attempts have been performed in order to quantify the energy transfer in planetary mills. For instance, models based on a kinematic approach [24-26] adequately describe the trajectory of a ball inside the vial of a planetary mill and suppose that collisions are the primary energy-transferring event. The energy of the impact is determined by the kinetic energy difference between the moment at which the ball detaches from the vial and when the ball, after flying at a constant speed, again reaches the surface of the vial and remains in contact with it. After each collision, the ball and the vial move jointly until the subsequent detachment. The total energy transferred to the powder mixture is then defined by the collision energy, the collision frequency and the milling time.

During a milling process involving a number of balls, the motions of the balls interfere with each other, and the total amount of energy transferred by the balls to the powder is not equivalent to the cumulative effect of the recurrent impacts of a single ball on the vial wall. To account for this situation, the models introduce a parameter related to the filling of the vial. However, when several balls are put in a vial, successive small collisions with other balls, the lack of single ball-wall collisions, and the slipping of balls are produced, so that the real situation approaches a chaotic system [28]. Recently, it has shown that the ball motion in planetary mills recorded with a high-speed camera differs significantly from ball trajectories calculated using kinematic equations [29]. Therefore, the trajectory of a ball in such a situation is difficult to describe and energy calculations that assume well-defined trajectories and ball-wall collisions can be inaccurate, although correction parameters are introduced. 
In the previous section, it was shown that ignition time is sensitive to variations in the mechanical dose of planetary mills induced by changes in the experimental conditions. Moreover, the dependence of the ignition time on the milling parameters allows the inference of a direct relationship between the inverse of the ignition time and the power delivered by a planetary mill. For these reasons, variation of the ignition time could be used to assess the theoretical models proposed in the literature to describe the energy transferred by a mill during operation. By way of example, the model of Burgio et al [24] was chosen because it describes the energy supplied by a planetary mill using only analytical expressions without any numerical calculation so that it was possible to more easily compare the quantitative predictions of the model with our observational data.

Assuming that the total energy transferred by the planetary mill per gram of the $\mathrm{Zn} / \mathrm{Se}$ reactant mixture and required to induce ignition is a constant value, the Burgio model defines this amount of energy by the following expression:

$$
\frac{E_{T}}{g}=-N_{b} \varphi_{b} f_{b} K_{a} m_{b}\left\{\omega_{v}^{3}\left(r_{v}-d_{b} / 2\right) / \omega_{p}+\omega_{p} \omega_{v} R_{p}\right\}\left(r_{v}-d_{b} / 2\right) t_{i g} / m_{c h}=\operatorname{cte}\left(J^{-1}\right)
$$

Where $\mathrm{N}_{\mathrm{b}}$ is the number of balls; $\varphi_{\mathrm{b}}$ is a parameter that accounts for the degree of filling of the vial [24]; $\mathrm{f}_{\mathrm{b}}$ is the frequency with which the balls are launched against the opposite wall of the vial $\left(\mathrm{s}^{-1}\right)[24] ; \mathrm{K}_{\mathrm{a}}$ is a constant that accounts for the elasticity of collisions, and a value of 1 represents perfectly inelastic collisions; $m_{b}$ is the ball mass $(\mathrm{kg})$; $d_{b}$ is the ball diameter $(m)$; $\omega_{\mathrm{p}}$ is the plate spinning rate $\left(\mathrm{rad} \mathrm{s}^{-1}\right) ; \omega_{\mathrm{v}}$ is the vial spinning rate $\left(\mathrm{rad} \mathrm{s}^{-1}\right) ; \mathrm{R}_{\mathrm{p}}$ is the distance between the centre of the plate and the centre of the vial ( $m) ; r_{v}$ is the vial radius $(m)$; $t_{i g}$ is the ignition time measured $(\mathrm{s})$; and $\mathrm{m}_{\mathrm{ch}}$ is the mass of the powder charge $(\mathrm{g})$.

If the experimental values used in all of the different experiments shown in section 3.2 are introduced into the above expression, a constant value should be obtained if the model 
adequately describes the energy transfer in planetary mills. The obtained results are shown in Figure 8. The experimental data provide a good fit to the model at a high spinning rate. As the spinning rate is reduced, it seems necessary to provide more energy to induce ignition. This behaviour suggests that collisions are less effective at transferring energy as the spinning rate decreases; it is as if the collisions were less and less inelastic. This deviation of the model can be corrected if the constant that defines the inelasticity of collisions decreases with the spinning rate from $600 \mathrm{rpm}$. The large deviation observed for the experiment at $200 \mathrm{rpm}$ is a special case because at this low spinning rate the balls are not launched; friction is the main energy transfer process, and a collision model cannot accurately describe this situation.

For the experiments with different balls, the model describes the situation well for all three types of balls when using 5, 6 or 7 balls. For 3 and 4 balls, a high deviation was observed that was particularly pronounced for the 3-ball case. For three balls, WC balls appear to be the least effective grinding medium because the model is based on collisions, and in the case of three balls, the energy is mainly transferred by friction. The density of the grinding medium is not as crucial under a friction regime. These results were confirmed when the energy was plotted versus the BPR, and the data provided a good fit to the model, except for 3 (particularly) and 4 balls.

The results shown in Figure 8 suggest that the Burgio model provides a valuable description of the energy transfer in planetary mills in the regime of high milling intensity, where collisions are the main energy-transferring event. This model is valid for a broad range of milling operative conditions. However, under other experimental conditions, where other phenomena (such as friction) play a non-negligible role, the model does not provide a realistic description of energy transfer.

The results in Figure $\mathbf{8}$ also provide evidence of the sensitivity of the ignition time to the experimental conditions that determine the milling regime and the ability of $t_{i g}$ to 
differentiate these regimes properly. Moreover, ignition time can be a helpful parameter in the difficult task of the scaling-up of planetary ball mills [30], since it could also be used to experimentally validate the results based on computational simulations.

\subsection{Comparison of efficiencies of milling devices.}

The results shown in this work support the idea that the comparison of the ignition time of the same MSR process carried out in different milling devices can provide direct information about the efficiencies of mills. To illustrate this, the present results were compared with three works found in the literature, where the formation of $\mathrm{ZnSe}$ by MSR can be inferred: the work of Tschakarov et al. [15], in which a low energy vibratory mill was used, and ignition was observed after $312 \mathrm{~min}$ and two more recent works by Rafea [17] and Achimovicova et al [21], in which the formation of ZnSe in planetary mills took place after approximately 12-16 min and 15-18 min, respectively. Rafea [17] used a Retsch PM 100 planetary mill at $300 \mathrm{rpm}$, but unfortunately the BPR value was not provided. Achimovicova et al. [21] employed a Fritsch Pulverisette 6 planetary mill at $300 \mathrm{rpm}$ with a BPR of 78.3.

If the data from references 17 and 21 are compared to those of the present work, an ignition time of approximately 15 min was observed using higher spinning rate, but lower BPR ratios. This ignition time was found for a BPR value of 37 and a spinning rate of 400 $\mathrm{rpm}$, or a BPR of 19 and a spinning rate of $500 \mathrm{rpm}$. If it is supposed that all of these milling experiments were carried out under similar milling intensities, it can be deduced that approximately doubling the BPR value allows the reduction of the spinning rate of a planetary mill by $100 \mathrm{rpm}$. Comparing with the work of Tschakarov et al [15], a long ignition time of 300 min was observed in the present work when the milling regime in the planetary mill was dominated by friction. 


\section{Conclusions}

The formation of $\mathrm{ZnSe}$ from an equiatomic $\mathrm{Zn}$-Se mixture that undergoes an MSR process has been used as an ideal system to study the dependence of ignition time on the main milling parameters characterising milling intensity in planetary mills. It was shown that ignition time, which can be considered to be the critical time needed to absorb the constant amount of energy required to ignite the reactant mixture, is highly sensitive to the experimental conditions. A third power dependence between the inverse of the ignition time and the spinning rate $\left(1 / t_{\text {ig }} \propto\right.$ spinning rate $\left.^{3}\right)$, a linear dependence between the ignition time and the powder charge, an inverse relationship between the ratio of the densities of the grinding media and the ignition times $\left(\rho_{1} / \rho_{2} \approx t_{i g}{ }^{2} / t_{i g}{ }^{l}\right)$, and an inverse relationship between the ignition time and the $\mathrm{BPR}\left(1 / t_{\text {ig }} \propto B P R\right)$ were found.

Moreover, the direct relationship established between the inverse of the ignition time and the mechanical dose provided by the mill enables the comparison of efficiencies of milling devices. This is of great importance because the data obtained in different mills with respect to time are generally incomparable and, consequently, irreproducible if the current absolute energy input is not accurately considered. The use of a well-defined MSR process as a universal standard system for the efficiency of mills and the comparison of results should be considered by the mechanochemical community. The use of this standard process could be also used to validate models proposed in the literature for the description of the energy transfer in milling devices. This method is also of enormous significance for scaling-up purposes.

\section{Acknowledgments}

This work was supported by the common project Slovak Academy of Sciences (SAS)

- Spanish National Research Council (CSIC) No. 2009SK0002 and by the Spanish 
government under grant No. MAT2011-22981, which was financed in part by the European Regional Development Fund of 2007-2013. 


\section{References}

[1] M. Nakach, J.R. Authelin, A. Chamayou, J. Dodds, Comparison of various milling technologies for grinding pharmaceutical powders, Int. J. Miner. Process. 74 (2004) S173-S181.

[2] Handbook of Powder Technology, vol. 12 Particle Breakage, in: A.D. Salman, M. Ghadiri, M.J. Hounslow (Eds.), Elsevier B.V., Amsterdam, 2007.

[3] M. He, Y. Wang, E. Forssberg, Slurry rheology in wet ultrafine grinding of industrial minerals: a review, Powder Technol. 147 (2004) 94-112.

[4] J.M. Córdoba, M.J. Sayagués, M.D. Alcalá, F.J. Gotor, Nanostructured powders of niobium, tantalum, and hafnium carbonitrides synthesized by mechanically induced selfpropagating reaction, J. Am. Ceram. Soc. 90 (2007) 381-387.

[5] M.A. Avilés, J.M. Córdoba, M.J. Sayagués, M.D. Alcalá, F.J. Gotor, Mechanosynthesis of $\mathrm{Hf}_{1-\mathrm{x}} \mathrm{Zr}_{\mathrm{x}} \mathrm{B}_{2}$ solid solution and $\mathrm{Hf}_{1-\mathrm{x}} \mathrm{Zr}_{\mathrm{x}} \mathrm{B}_{2} / \mathrm{SiC}$ composite powders, J. Am. Ceram. Soc. 93 (2010) 696-702.

[6] L. Takacs, Self-sustaining reactions induced by ball milling, Prog. Mater. Sci. 47 (2002) $355-414$.

[7] M. Atzmon, In situ thermal observation of explosive compound-formation reaction during mechanical alloying, Phys. Rev. Lett. 64 (1990) 487-490.

[8] L. Takacs, V. Sepelak, Quantitative comparison of the efficiency of mechanochemical reactors, J. Mat. Sci. 39 (2004) 5487-5489.

[9] G.B. Schaffer, P.G. McCormick, On the kinetics of mechanical alloying, Metall. Trans. A 23 (1992) 1285-1290.

[10]C. Deidda, F. Delogu, F. Maglia, U. Anselmi-Tamburini, G. Cocco, Mechanical processing and self-sustaining high-temperature syntheis of TiC powders, Mat. Sci. Eng. A 375-377 (2004) 800-803. 
[11]G.B. Schaffer, J.S. Forrester, The influence of collision energy and strain accumulation on the kinetics of mechanical alloying, J. Mat. Sci. 32 (1997) 3157-3162.

[12]A. Bakhshai, V. Soika, M.A. Susol, L. Takacs, Mechanochemical reactions in the Sn-ZnS system: Further studies, J. Solid State Chem. 153 (2000) 371-380.

[13]L. Takacs, M.A. Susol, Gradual and combustive mechanochemical reactions in the SnZn-S system, J. Solid State Chem. 121 (1996) 394-399.

[14]G. Mulas, S. Loiselle, L. Schiffini, G. Cocco, The mechanochemical self-propagating reaction between hexachlorobenzene and calcium hydride, J. Solid State Chem. 129 (1997) 263-270.

[15]CH.G. Tschaharov, G.G. Gospodinov, Z. Bontschev, Über den Mechanismus der mechanochemischen Synthese anorganischer Verbindungen, J. Solid State Chem. 41 (1982) 244-252.

[16]M.A. Susol, MS thesis, University of Maryland, Baltimore County, 1995.

[17]M.A. Rafea, Preparation and characterization of ZnSe nanoparticles by mechanochemical process, J. Mater. Sci.: Mater. Electron. 18 (2007) 415-420.

[18]J. Che, X. Yao, X. Wan, H. Jiang, M. Wang, Synthesis of ZnSe nanocrystalline powders by mechanochemical reaction, J. Electroceram. 21 (2008) 729-732.

[19] J. Li, M. Wang, X. Huo, X. Yao, Preparation and optical properties of dispersible ZnSe nanocrystals synthesized by high energy ball milling, Ceram. Int. 34 (2008) 1077-1080.

[20]J. Baltazar-Rodrigues, J.C. de Lima, C.E.M. Campos, T.A. Grandi, Temperature effects on mechanically alloyed nanometrric ZnSe powder, Powder Technol. 189 (2009) 70-73.

[21]M. Achimovičová, P. Baláž, T. Ohtani, N. Kostova, G. Tyuliev, A. Feldhoff, V. Šepelák, Characterization of mechanochemically synthesized $\mathrm{ZnSe}$ in a laboratory mill and an industrial mill, Solid State Ionics 192 (2011) 632-637. 
[22]Y.H. Zhao, Y.T. Zhu, T. Liu, Mechanism of solid-state amorphization of Se induced by mechanical milling, J. Appl. Phys. 95 (2004) 7674-7680.

[23] M. Binnewies, E. Milke, Thermochemical data of elements and compounds, Wiley-VCH, Weinheim, 1999.

[24] N. Burgio, A. Iasonna, M. Magini, S. Martelli, F. Padella, Mechanical alloying of the FeZr system: Correlation between input energy and end products, Il Nuovo Cimento 13 (1991) 456-476.

[25] M. Magini, A. Iasonna, F. Padella, An experimental support to the energy transfer evaluated by the collision model, Scrip. Mater. 34 (1996) 13-19.

[26]M. Abdellaoui, E. Gaffet, The physics of mechanical alloying in a planetary ball mill: Mathematical treatment, Acta Metall. Mater. 43 (1995) 1087-1098.

[27]C. Cuadrado-Laborde, L.C. Damonte, L. Mendoza-Zélis, Ball milling sound statistical analysis and its relationship with the mechanical work intensity, Rev. Sci. Instrum. 75 (2004) 2334-2339.

[28]P. Le Brun, L. Froyen, L. Delaey, The modelling of the mechanical alloying process in a planetary ball mill: comparison between theory and in-situ observations, Mat. Sci. Eng. A $161(1993) 75-82$.

[29] S. Rosenkranz, S. Breitung-Faes, A. Kwade, Experimental investigations and modelling of the ball motion in planetary ball mills, Powder Technol. 212 (2011) 224-230.

[30]H. Mio, J. Kano, F. Saito, Scale-up method of planetary ball mill, Chem. Eng. Sci. 59 (2004) 5909-5916. 


\section{Figure captions}

Figure 1. Modified planetary ball mill Pulverisette 7 (Fritsch) employed in this study allowing operation at a constant gas pressure and the detection of self-sustaining reactions during milling.

Figure 2. X-ray powder diffraction diagrams of an equiatomic $\mathrm{Zn} / \mathrm{Se}$ mixture milled for increasing times: (ם) Se; (○) Zn; (O) ZnSe (cubic); ( $\square$ ) ZnSe (hexagonal).

Figure 3. DSC measurements of an equiatomic $\mathrm{Zn} / \mathrm{Se}$ mixture milled for increasing times.

Figure 4. (a) Dependence of the ignition time on the spinning rate of the planetary mill. (b) Linear relationship between $1 / \mathrm{t}_{\mathrm{gg}}$ and the third power of the spinning rate.

Figure 5. Dependence of the ignition time on the powder charge.

Figure 6. Dependence of the ignition time on the number and type of balls.

Figure 7. Dependence of the ignition time on (a) the BPR, and (b) the PBR.

Figure 8. Energy per gram of the equiatomic $\mathrm{Zn} / \mathrm{Se}$ reactant mixture supplied by the planetary mill to induce ignition as a function of (a) the spinning rate, (b) the number of balls, and (c) the BPR, according to the model of Burgio et al. [21]. 


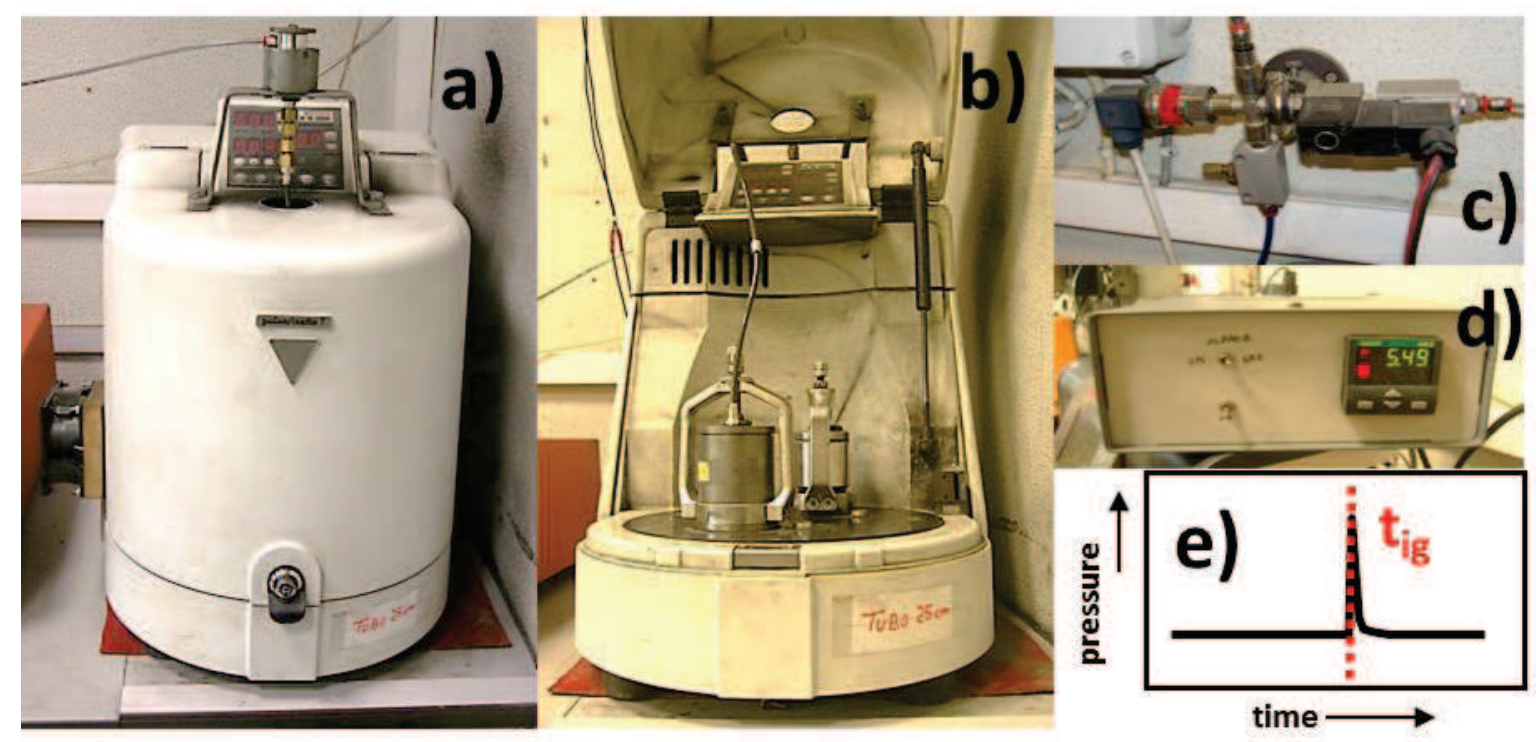

FIGURE 1 


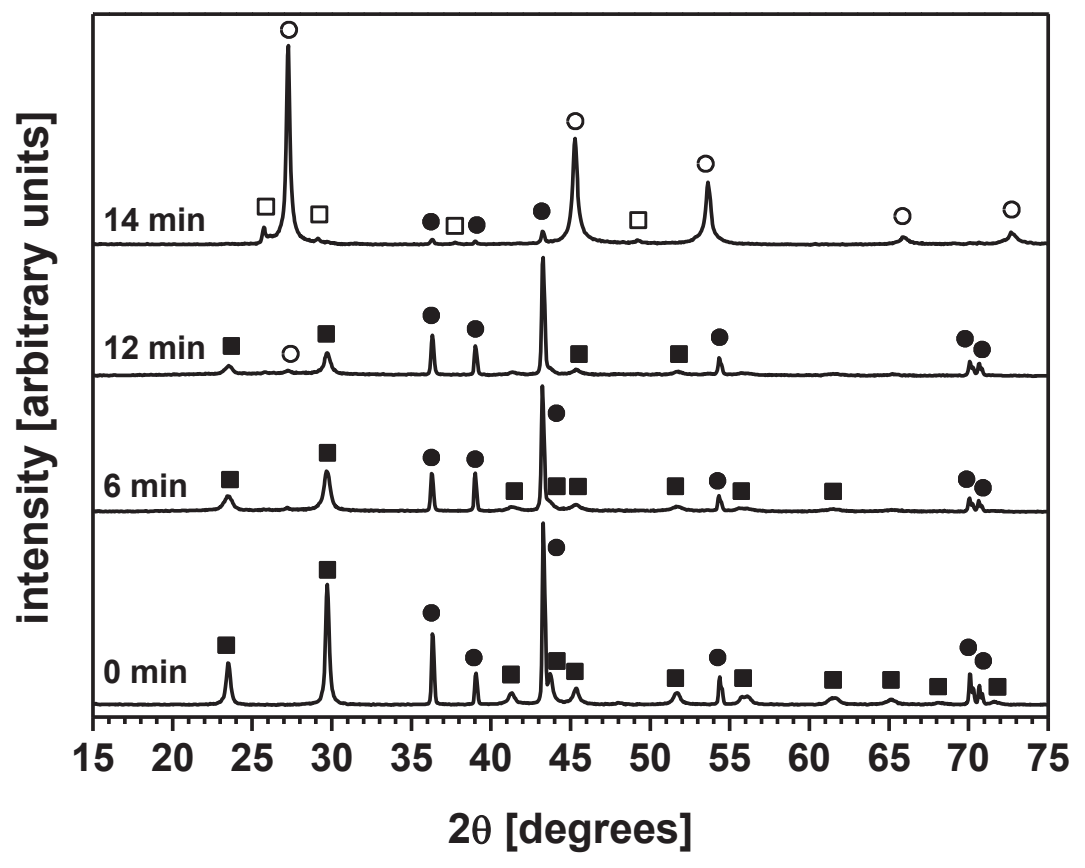

FIGURE 2 


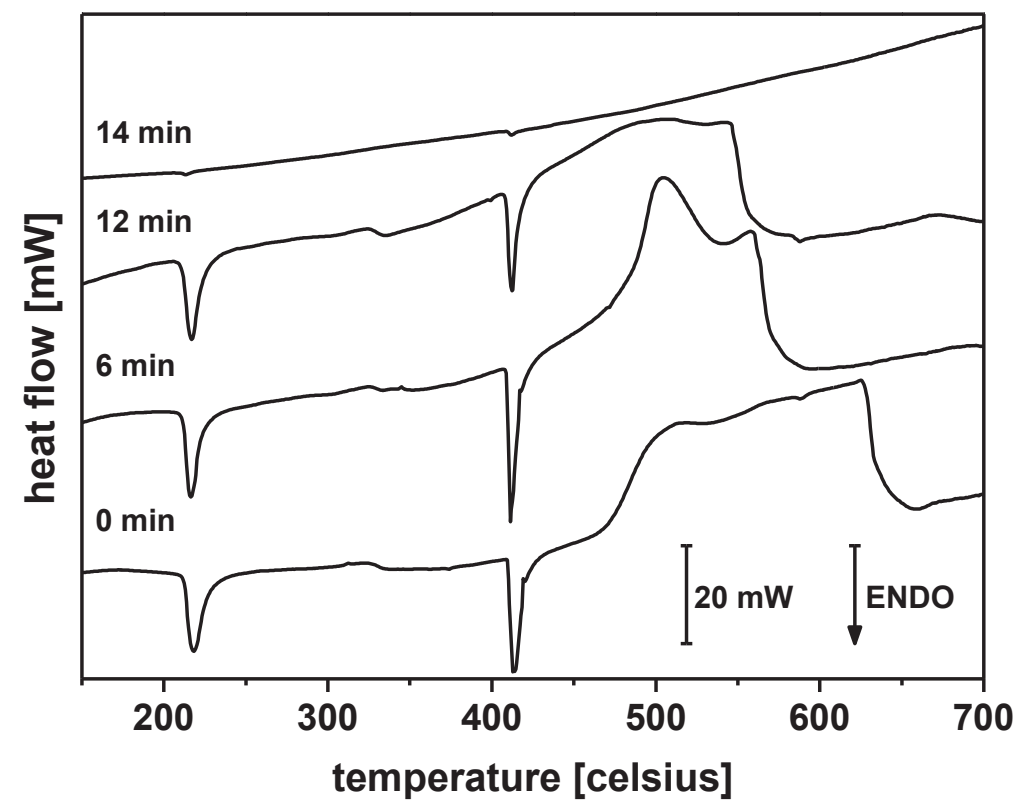

FIGURE 3 

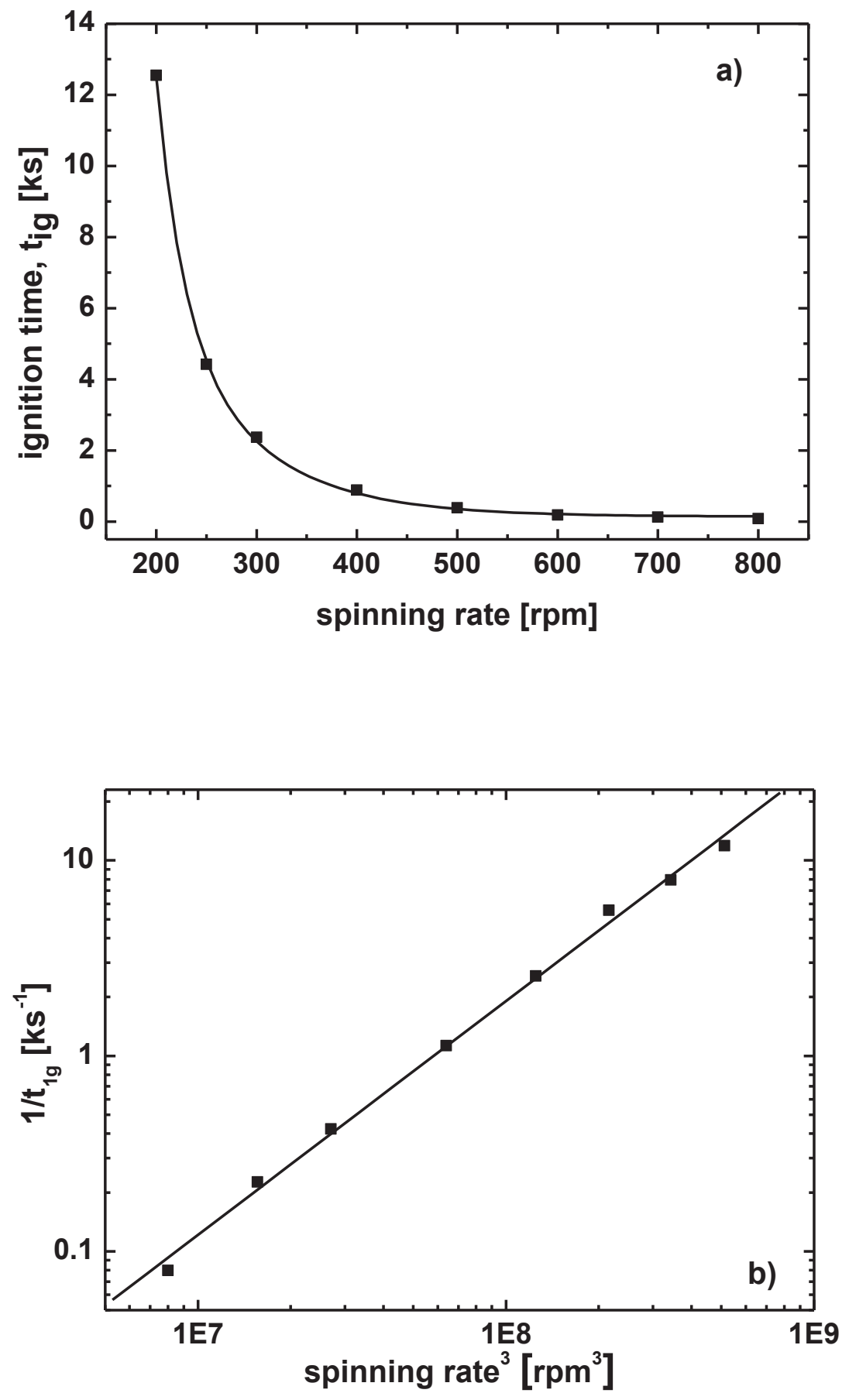

FIGURE 4 


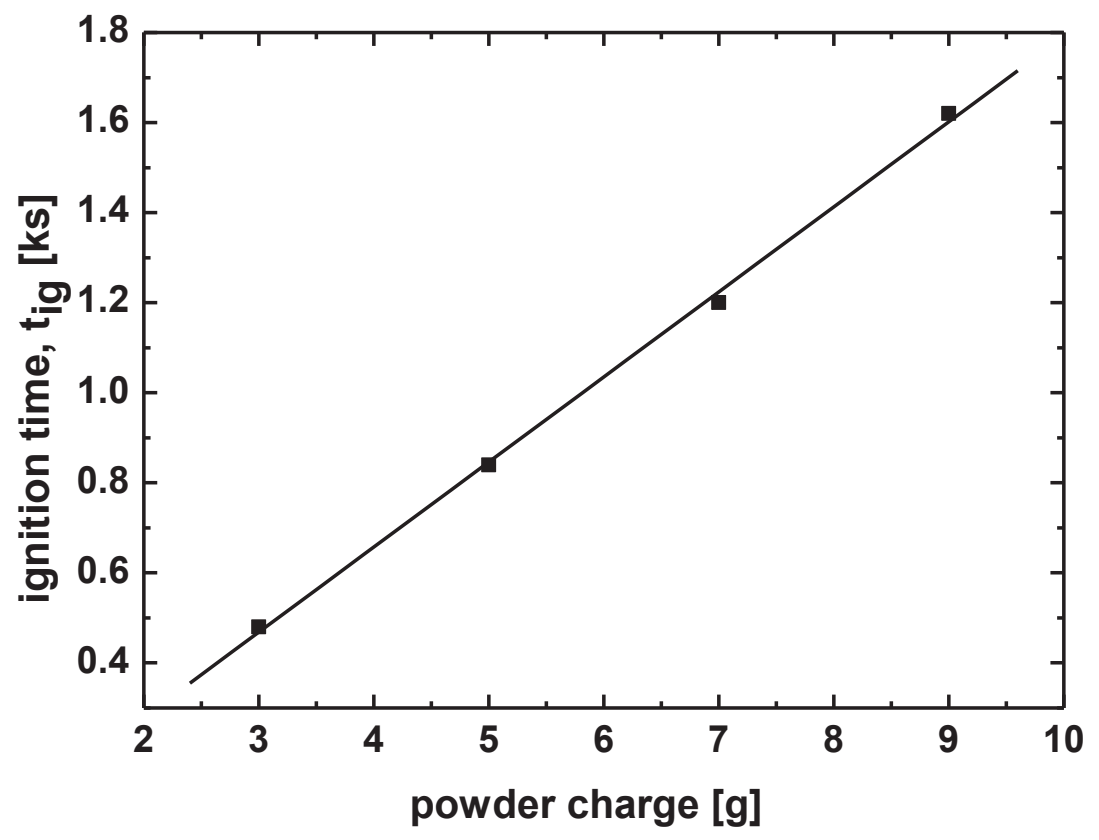

FIGURE 5 


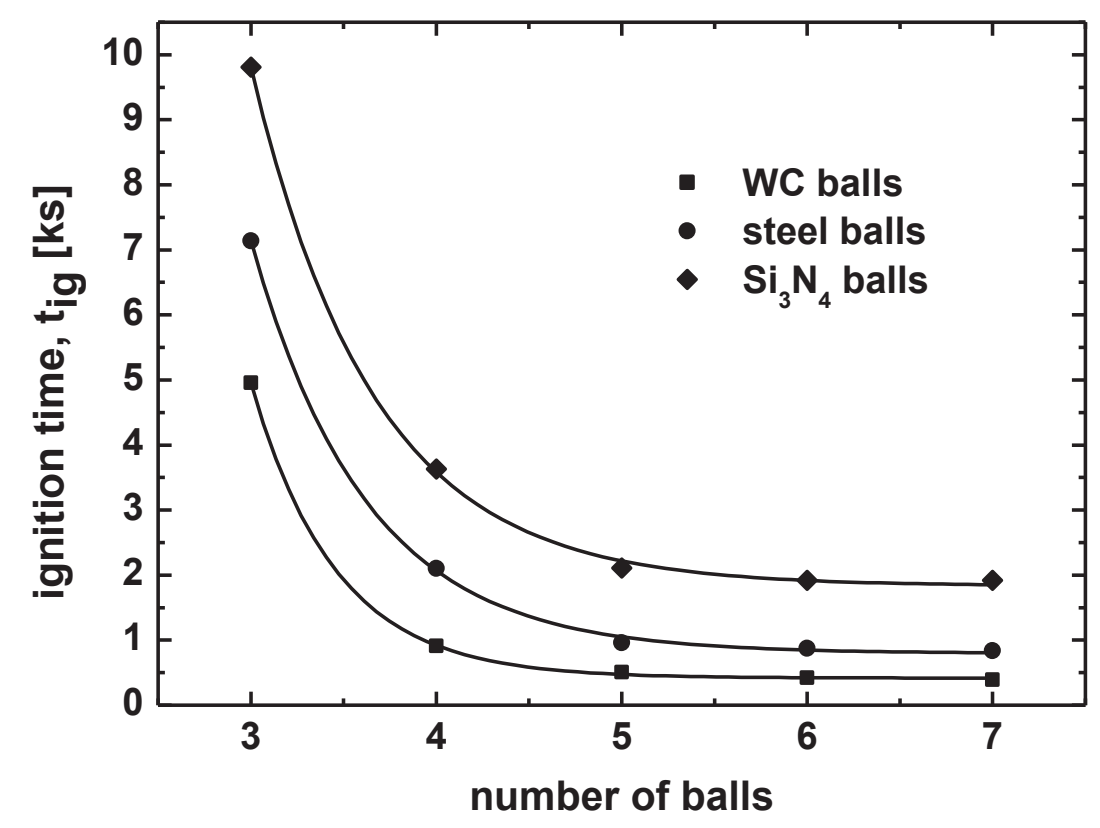

FIGURE 6 

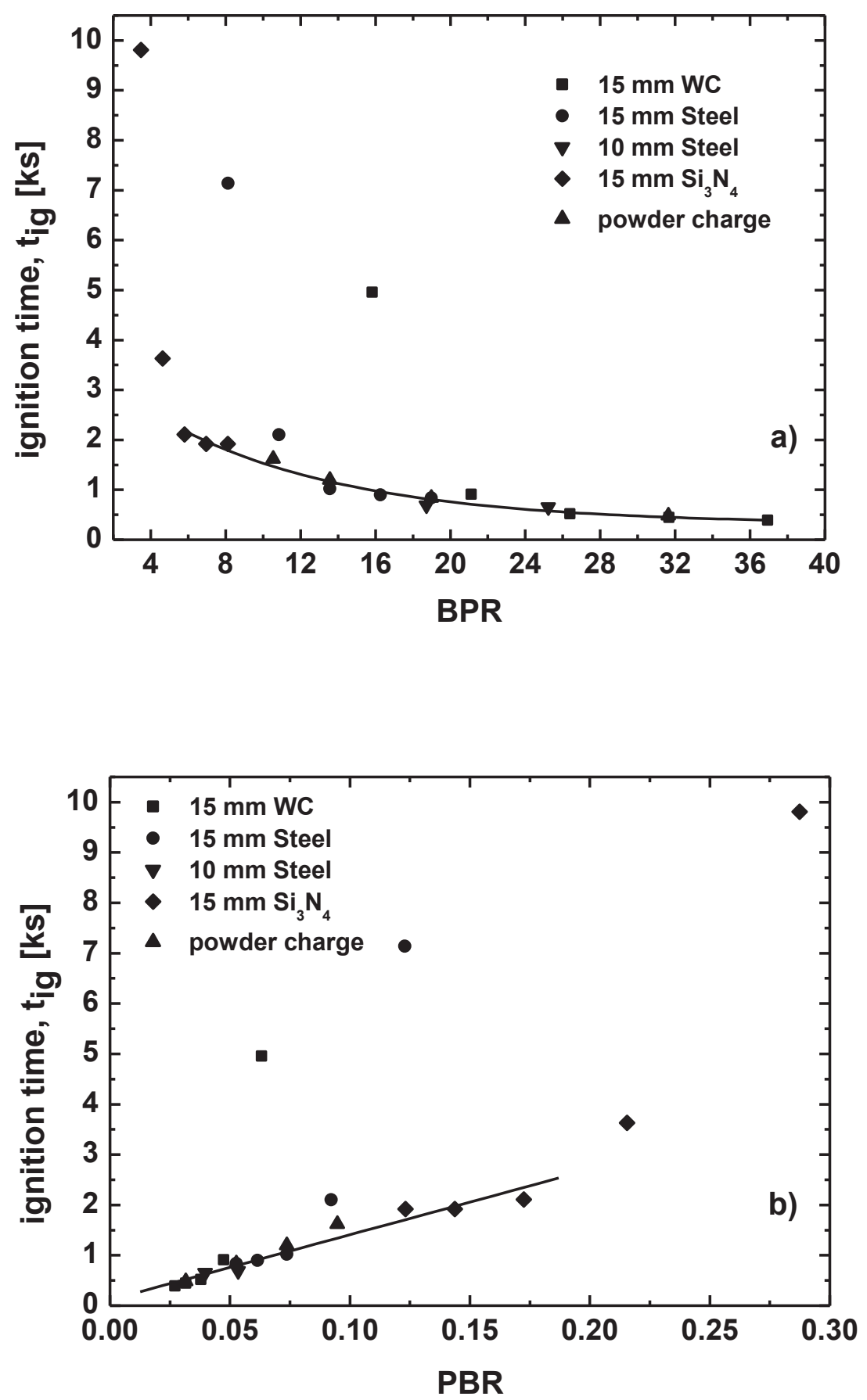

FIGURE 7 

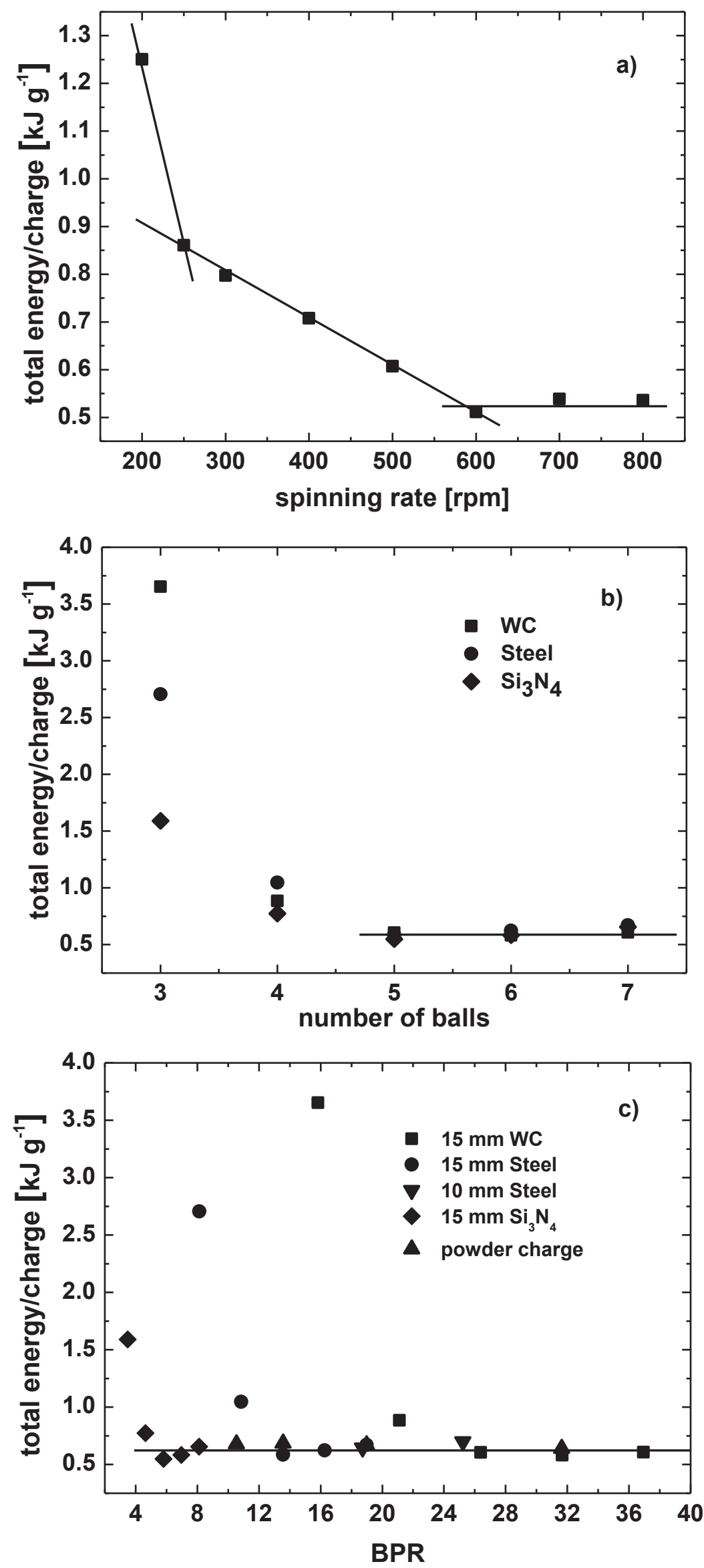

FIGURE 8 